\title{
Effect of vanillic acid on pentylenetetrazole-kindled rats: Nrf2/HO-1, IGF-1 signaling pathways cross talk
}

Vanillic acid (VA) exhibited antioxidant and neuroprotective properties in some neurodegenerative disorders. So, the current study examined the neuroprotective potential of VA as an antiepileptic agent in pentylenetetrazole (PTZ)-induced epileptic rats and the prospective role of Insulin like growth factor-1 (ICF-1) and nuclear factor-2 erythroid-related factor-2 (Nrf2)/heme oxygenase-1 (HO-1) pathway in this respect. Thirty male albino rats were equally subdivided into 3 groups; (1) normal control (NC) group, (2) PTZ-group: received PTZ (50 mg/Kg, i.p. every other day) for 14 days, and (3) PTZ + VA group: received PTZ and VA ( $50 \mathrm{mg} / \mathrm{Kg}$ daily for 2 weeks). The seizure score and latency were evaluated after PTZ injection. Also, the markers of oxidative stress (malondialdehyde (MDA), catalase, and reduced glutathione (CSH)), histopathological examination, the expression of glial fibrillary acidic protein (GFAP) (a marker of astrocytes) ICF1, Nrf2, and HO-1 were assessed in the brain tissues by the end of the experiment. PTZ caused significant decrease in seizure latency and significant increase in seizure score by the end of the experiment $(p<0.01)$. This was associated with significant increase in MDA and GFAP with significant decrease in CSH, total antioxidant capacity (TAC) and IGF- 1 in brain tissues compared to normal group $(p<$ 0.01 ). On the other hand, treatment with VA caused significant attenuation in PTZ-induced seizures which was associated with significant improvement in oxidative stress markers and downregulation in GFAP and upregulation of Nrf2, HO-1 and IGF-1 in CA3 hippocampal region $(p<0.01)$. VA showed neuroprotective and anti-epileptic effects against PTZ-induced epilepsy which probably might be due to its antioxidant properties and upregulation of $\mathrm{Nrf} 2 / \mathrm{HO}^{-1}$ pathway and IGF-1.

Keywords

PTZ-induced epilepsy; Vanillic acid; ICF-1; Nrf2; HO-1

\section{Introduction}

Epilepsy is a serious neurological disorder that impacts about $1 \%-2 \%$ of the world's population [1]. Epilepsy is accompanied with cognitive deficits which negatively influence life's quality [2] and about one third of patients with epilepsy show resistance to the established lines of treatment [3]. So, looking into the possible mechanisms of the process of epileptogenesis is mandatory to develop new agents that regulate epileptic seizures during epilepsy. During the period of epileptogenesis, molecular events bring about various changes in the brain structure including loss of neurons, sprouting of mossy fibers, reorganization of synapses, astrocytosis, neurogenesis, etc. [4]. The process of epileptogenesis can be replicated through kindling. Kindling is a process through which we can ignite prolonged seizures with gradually increased duration and degree of behavioral disorder. Kindling is achieved by repetitive sub-threshold applications of any convulsing agent. Chemical kindling via pentylenetetrazole (PTZ) is one way to induce animal models for temporal lobe epilepsy, which is the most common symptomatic refractory form of epilepsy [5]. Oxidative stress is one of the major mechanisms which initiates epilepsy and leads to its progression next to a primary brain insult [6]. Oxidative stress in neurons during epilepsy results from excessive production of reactive oxygen radicals (ROS), apoptotic, inflammatory, immune changes and dysfunction of bloodbrain barrier $[7,8]$. So, the use of antioxidants as ascorbic acid [9], flavonoids [10], vitamin E [11], L-carnitine [12] etc., protected against epilepsy in animal models.

Nuclear factor-2 erythroid-related factor-2 (Nrf2) is a transcription factor that promotes antioxidant enzymes production such as heme oxygenase-1 (HO-1), superoxide dismutase (SOD) glutathione peroxidase (GPx), thus renders cells oxidative stress resistant [13]. Nrf2 is the principal transcription factor activated succeeding oxidative stress in epilepsy representing an endogenous adaptive mechanism that protect against the neuronal oxidant insult [14]. In case of temporal lope epilepsy, Nrf2/antioxidant response element (ARE)-dependent $\mathrm{HO}-1 / \mathrm{NAD}(\mathrm{P}) \mathrm{H}$ dehydrogenase (quinone)-1 (NQO-1) production was found to ameliorate 
oxidative injury induced by glutamate. Nrf2/HO-1 pathway activation can be considered a potential therapeutic avenue in intractable epilepsy $[13,15]$. Moreover, IGF-1 refines the central nervous system development, aids neurons to differentiate, mature and connect properly. It urges axons to grow and neurons to survive [16]. Insulin like growth factor-1 (IGF-1)/phosphoinositide 3-kinase (PI3K)/protein kinase B (Akt) pathway has been recently featured as a protective pathway against oxidative stress in astrocytes. It was also implicated in ameliorating neurodegenerative changes in Alzheimer's [17]. Recently, it has been found that IGF-1 induce a transcriptional program for mitochondrial biogenesis through induction of Nrf2 expression [18]. So, we suggest that there would be a cross talk between IGF-1 and Nrf2/HO1 pathways.

Plant phenolics including vanillic acid (VA), the major constituent of vanilla bean and pod extracts [19], are commonly used as a food flavour. It has been reported that they have neuroprotective properties and can be used in treatment of several disorders [20]. Also, vanillic acid demonstrated antioxidative, antihypertensive and anti-inflammatory properties [21-23]. Recently, VA was reported to activate the Nrf2/HO-1 pathway in neurodegenerative disorders as Alzheimer's disease. Also, VA exerted its antioxidant effect through the protein kinase $\mathrm{B}(\mathrm{Akt}) / \mathrm{glycogen}$ synthase kinase $3 \beta$ (GSK $3 \beta$ )/Nrf2 signaling pathway in mice brains [24]. We hypothesized that VA could attenuate epileptic seizures induced by PTZ in rats via Nrf2/HO-1 and IGF-1 activation. So, the present study aimed to investigate the impact of VA on PTZ-induced epileptic seizure and to explore the role of $\mathrm{Nrf2/HO}-1$ and IGF-1 in this possible neuroprotective of VA in rats.

\section{Materials and methods}

\subsection{Experimental animals}

Thirty male sprague-dawely rats weighs 170-190 g were accommodated in standard cages at Medical Experimental Research, Mansoura, Egypt. Animals fed on standard diet and water adlibitum. Animal care was done according to The Care and Use of Laboratory Animals (1996, published by National Academy Press, 2101 Constitution Ave. NW, Washington, D.C. 20055, USA). The IRB-committee approved all experimental procedures (code \#R.20.11.1098.r).

\subsection{Study design}

Rats were randomly (sealed envelopes) partitioned into 3 equal groups (10 rats in each) as the following: (a) Normal group: normal animals received $0.2 \mathrm{~mL}$ saline via intraperitoneal injection (i.p.) for 2 weeks, (b) PTZ group: rats were i.p injected with $50 \mathrm{mg} / \mathrm{kg}$ PTZ (purchased from Sigma Aldrich, USA) in $0.2 \mathrm{~mL}$ every other day for 2 weeks [25] and (c) VA + PTZ group: the same as PTZ group except that rats were pretreated with VA (purchased from Sigma Aldrich, USA) daily $50 \mathrm{mg} / \mathrm{kg}$ VA by oral gavage $30 \mathrm{~min}$ prior to PTZ for 2 weeks [22].

\subsection{Animal model}

Subsequent to each PTZ injection, rats were placed in transparent Plexiglas cages to record their 30 min convulsive behavior by a video camera after PTZ administration for 2 weeks or 7 records or trials (every other day). Latency to seizure onset $(\mathrm{sec})$ and seizure score were recorded. Scoring of seizure severity was done based on Racine's scale (0 = normal, non-epileptic activity, $1=$ movements of mouth and face, hyperactivity, grooming, sniffing, scratching, wet dog shakes, 2 = head nodding, staring, tremor, 3 = forelimb clonus, forelimb extension, 4 = rearing, salivating, tonic clonic activity and $5=$ falling, status epilepticus) [26].

\subsection{Harvesting of brain tissues}

At the end of the study, a high dose of $\mathrm{Na}^{+}$thiopental (120 $\mathrm{mg} / \mathrm{kg}$ ) i.p. was used for rat sacrifice. Six rats were perfused with one hundred $\mathrm{mL}$ heparinized saline via cardiac catheter for brain collection for biochemical and molecular studies, while the rest of rats (4 rats in each group) were perfused with one hundred $\mathrm{mL}$ heparinized saline then one hundredand fifty-mL formalin (10\%) through a cardiac catheter for collection of brains for histopathological and immunohistochemical studies. Details of collection and storing of brain tissues is described in our previous work [12].

\subsection{Assay of markers of oxidative stress (MDA, catalase activity, total antioxidant capacity) in brain tissues}

Hippocampal regions of the brain were homogenized in 1-2 mL cold phosphate buffered saline (50 mM in EDTA (1 $\mathrm{mM})$ at $\mathrm{pH}$ 7.5. Then, centrifugation done at $4000 \mathrm{rpm}$ at 4 ${ }^{\circ} \mathrm{C}$ for $15 \mathrm{~min}$. Colorimetric assay of these markers were done by using commercially available kits (Bio-Diagnostics, Dokki, Giza, Egypt) according to the manufacturer's instructions.

\subsection{Hematoxylin and eosin staining}

Brain tissues ( $n=4$ rats in each group) staining with hematoxylin and eosin (H\&E) was achieved based on our preceding work [12]. Neuronal loss and pyknotic nuclei were assessed by light microscopy in the CA3 region of hippocampus [12].

2.7 Assessment of Nrf2 and HO- 1 expression at the level of mRNA by real-time polymerase chain reaction $(P C R)$

The mRNAs encoding for antioxidant transcription factor, Nrf2 and heme oxygenase (HO)-1 were identified by real-time PCR in brain tissues. According to the manufacturer's instructions, we isolated the total RNA from brain tissue specimens. RNA was quantified spectrophotometrically, and its quality was determined by agarose gel electrophoresis and ethidium bromide staining. cDNA was synthesized from $1 \mu \mathrm{g}$ total RNA and then buffered in a volume of $25 \mu \mathrm{L}$. Then, the $25 \mu \mathrm{L}$ cDNA was diluted in a total volume of $100 \mu \mathrm{L}$. All details of gene (Nrf2, HO-1 and GAPDH) amplification and detection were mentioned in our previous work [27].

\subsection{Immunohistochemical examination for glial fibrillary acidic protein (GFAP), and IGF-1 in cornu ammonis (CA) CA3 and CA1 regions of the hippocampus}

Sagittal section $(40 \mu \mathrm{m})$ was obtained from brain tissue ( $\mathrm{n}=4$ rats in each group) to perform immunohisto- 

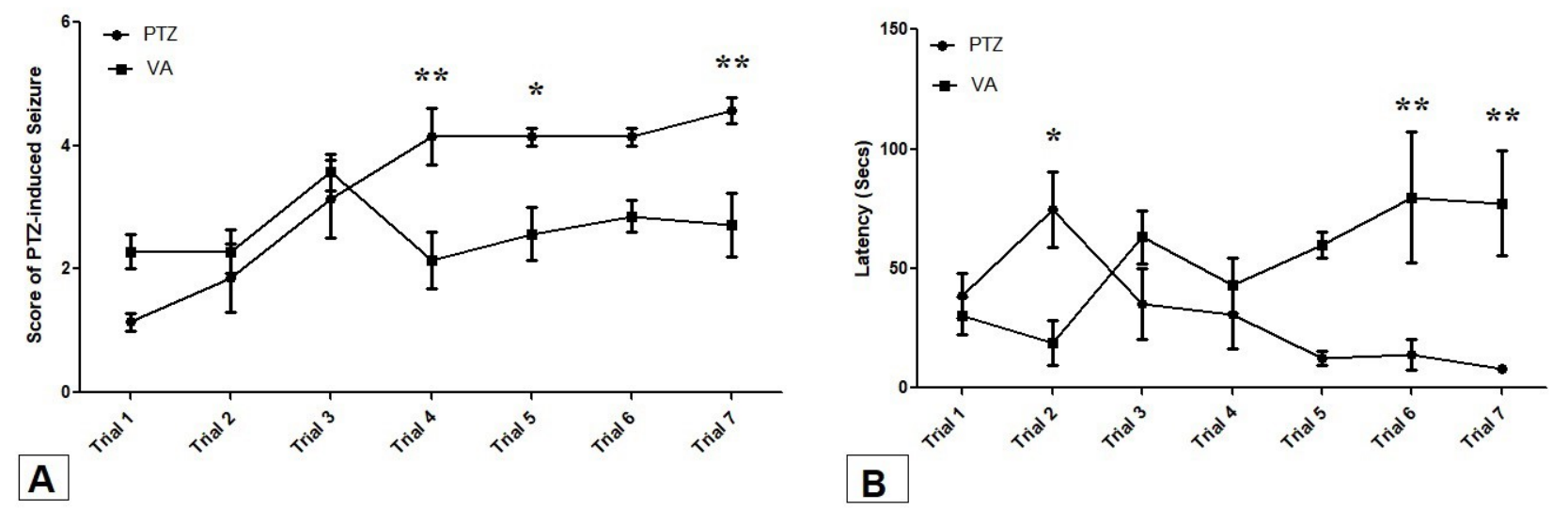

Fig. 1. Effects of VA on behavioral effects in PTZ-induced seizures. (A) seizure score. (B) seizure latency (Sec). Two-way ANOVA test, results were represented as mean \pm SEM. ${ }^{* *}$ significant vs PTZ group $p<0.001$ and *significant vs PTZ group $p<0.01$.
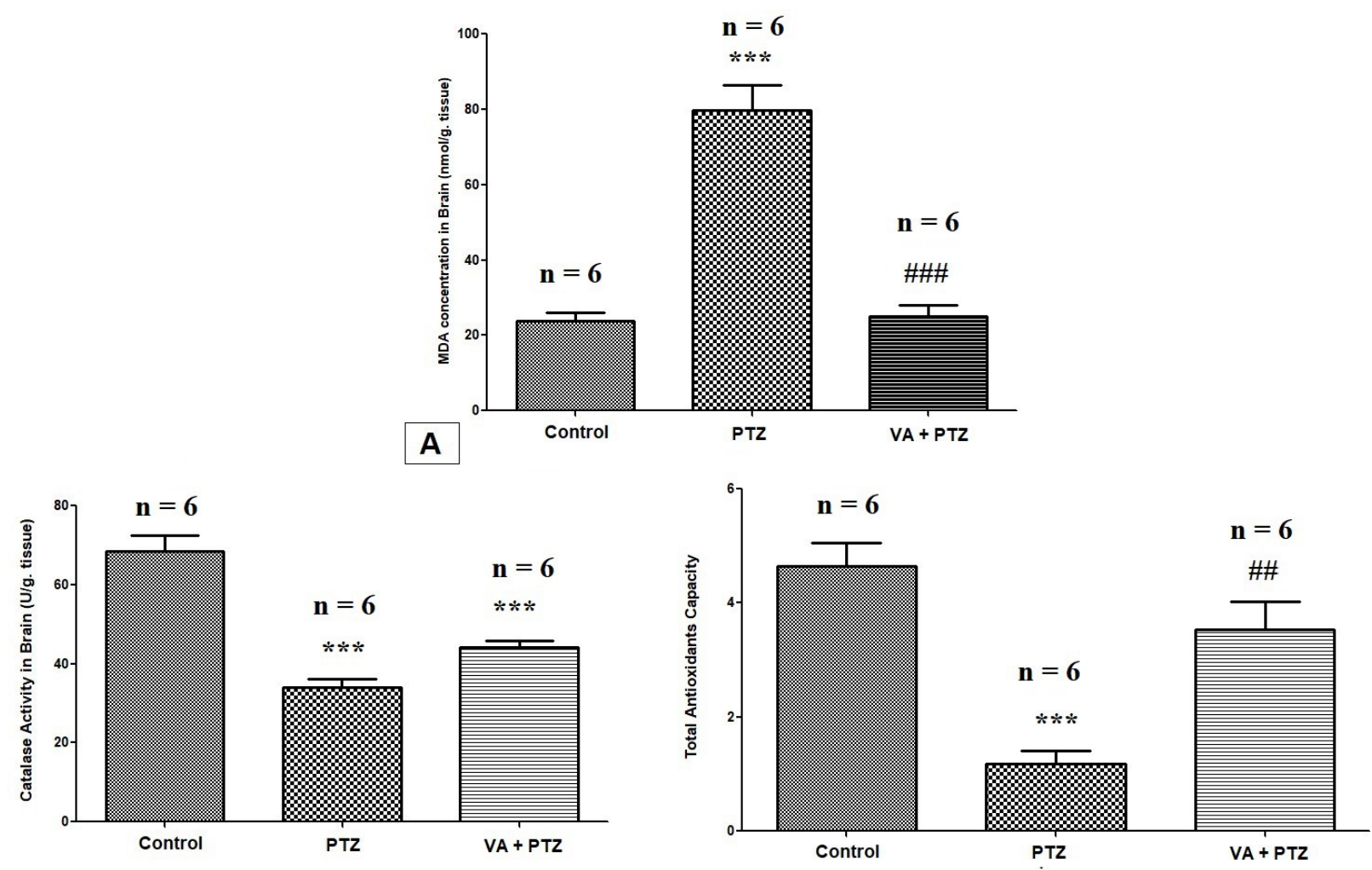

B

C

Fig. 2. Effects of VA on oxidative stress markers in PTZ-induced seizures. (A) Malondialdehyde (MDA) (nmol/g brain tissues). (B) Catalase (CAT) enzyme activity (U/g brain tissues). (C) Total antioxidant capacity (TAC). One-way ANOVA test, results were represented as mean \pm SEM. ${ }^{* * *}$ significant vs control group $p<0.0001,{ }^{\# \# \#}$ significant vs PTZ group $p<0.0001$ and ${ }^{\# \#}$ significant vs PTZ group $p<0.001 . \mathrm{n}=$ number of rats per group.

chemical staining based on a prior work [28]. The primary rabbit anti-glial fibrillary acidic protein (GFAP) antibody (cat\#SAB5600060, 1:200, Sigma, USA), and IGF-1 rabbit anti-rat monoclonal antibodies (Cat\# sc-74116, 1:50, Santa Cruz Biotechnology Inc., Santa Cruz, CA, USA) (with final dilution 1:50) were used. The hippocampus was examined blindly by 2 expert pathologists using optika light microscope with optika B-10 (OPTIKA Microscopes, Italy) and the
CA3 hippocampal region was analyzed for the immunostaining of these markers and the average of the number of GFAPpositive cells and region of interest of interest (ROI) of IGF-1 membranous immunostaining in 5 high power fields (HPF) were calculated. 


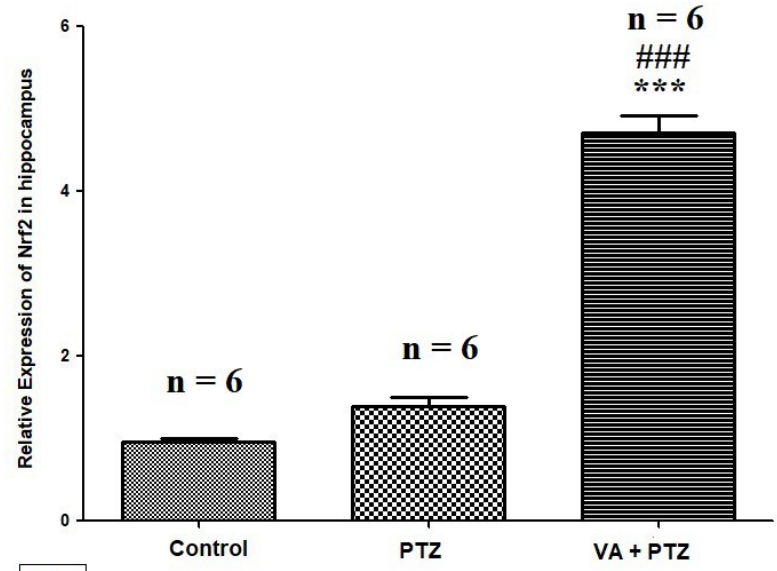

A

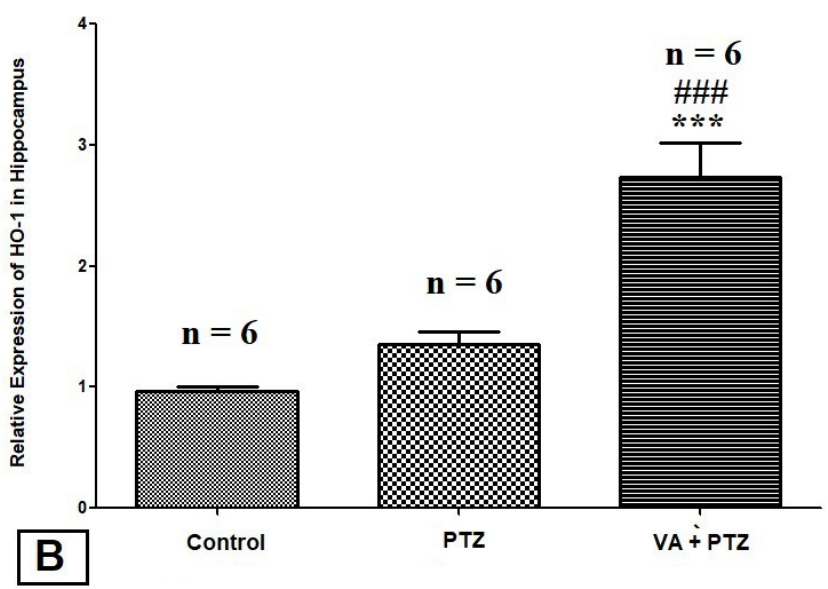

Fig. 3. Effects of VA in brain tissues. (A) The expression of Nrf2. (B) The expression of heme oxygenase (HO)-1. Two-way ANOVA test, results were represented as mean \pm SEM. ${ }^{* * *}$ significant vs control group $p<0.0001$ and ${ }^{\# \# \#}$ significant vs PTZ group $p<0.0001$. $\mathrm{n}=$ number of rats per group.

\subsection{Statistical analysis}

GraphPad Prism version 5.0 (GraphPad Software Inc., CA, USA) was used for statistical analysis. Statistical significance in behavioral parameters was determined by two-way analysis of variance (ANOVA) with Bonferroni posthoc test. Also, one-way ANOVA with Tukey's posthoc test was applied to find the statistical significance in biochemical, molecular, and histochemical parameters. Also, Pearson correlations between IGF-1 expression and other studied parameters were calculated. Statistical significance is determined when $p \leq 0.05$.

\section{Results}

3.1 VA effect on seizure latency and score in PTZ-induced epilepsy

In comparison with PTZ group, rats with VA administration displayed significant reduction in the PTZ-induced seizure score in trial $4(p<0.01)$, trial $5(p<0.05)$ and trial 7 ( $p<0.001)$ (Fig. 1A). Also, the latency of epileptic seizures showed significantly longer values in the VA group compared to the PTZ group in trials $(2,6,7)(p<0.05)$ (Fig. 1B).

\subsection{Effects of VA on MDA, catalase activity and total antioxidant} capacity (TAC) in brain tissues of PTZ-induced epilepsy

The concentration of MDA in the brain tissues showed a significant increase in the PTZ group as opposed to the control group $(p<0.0001)$. However, the VA group exhibited a significant reduction in the MDA concentration in comparison to the PTZ group $(p<0.01)$. On the other hand, catalase enzyme activity revealed a significant reduction in the PTZ group as opposed to the control group $(p<0.0001)$, while rats with VA showed a non-significant rise in the catalase (CAT) activity as opposed to the ones received PTZ only. Also, the total antioxidant capacity (TAC) showed a significant drop in rats with $\mathrm{PTZ}$ in contrast with the control rats $(p<0.001)$ while, VA administration displayed significantly increased TAC in comparison with PTZ only $(p<0.001)$ (Fig. 2A-C).

\subsection{VA effect on Nrf2 and HO-1 expression at the level of $m R N A$ in rat hippocampus}

In the hippocampus, the Nrf2 and $\mathrm{HO}-1$ expression at the mRNA level showed non-significant increase in the PTZ rats as opposed to the control ones $(p>0.05)$. However, rats with VA exhibited significantly elevated Nrf2 and HO-1 in comparison to the ones with PTZ only $(p<0.001)$ (Fig. 3A-B).

\subsection{VA effect on hippocampal histopathology}

Control rats displayed normally shaped neurons with normal number in the hippocampal CA3 subfield with histopathological examination (Fig. 4A-B), while neurons in the $\mathrm{CA} 3$ region in rats with $\mathrm{PTZ}$ were irregularly arranged, of diminished number and showed pyknosis (darkly stained nucleus and cytoplasm) (Fig. 4C-D). However, VA expanded the territory of normal neurons and decreased the number of abnormal ones in the hippocampus CA3 region (Fig. 4E-F).

\subsection{Effects of VA on GFAP and IGF-1 expression by immunohistochemistry in the hippocampus $C A 3$ region}

Fig. 5A displays a significant rise in the GFAP positive cells number (a marker of astrocytosis) in the hippocampus CA3 subfield in the PTZ group as opposed to the control one ( $p$ $<0.0001$ ). In contrast, VA significantly lowered the positive cells number in comparison to the PTZ group $(p<0.001)$. Brain specimens from different groups showed mild expression of astrocytes by GFAP in the control rats (Fig. 5B), substantial expression of GFAP in rats with PTZ (Fig. 5C), and mild expression of GFAP in VA group (Fig. 5D).

Moreover, the ROI of IGF-1 positivity in the hippocampus CA3 region significantly decreased in the PTZ group as opposed to the control one $(p<0.001)$. Moreover, VA significantly elevated the IGF-1 expression in comparison to the PTZ group ( $p<0.01$ ) (Fig. 6A). Brain specimens obtained showed marked cytoplasmic expression of IGF-1 in the control group (Fig. 6B), mild cytoplasmic expression in the PTZ group (Fig. 6C), moderate cytoplasmic expression in the VA group (Fig. 6D). 

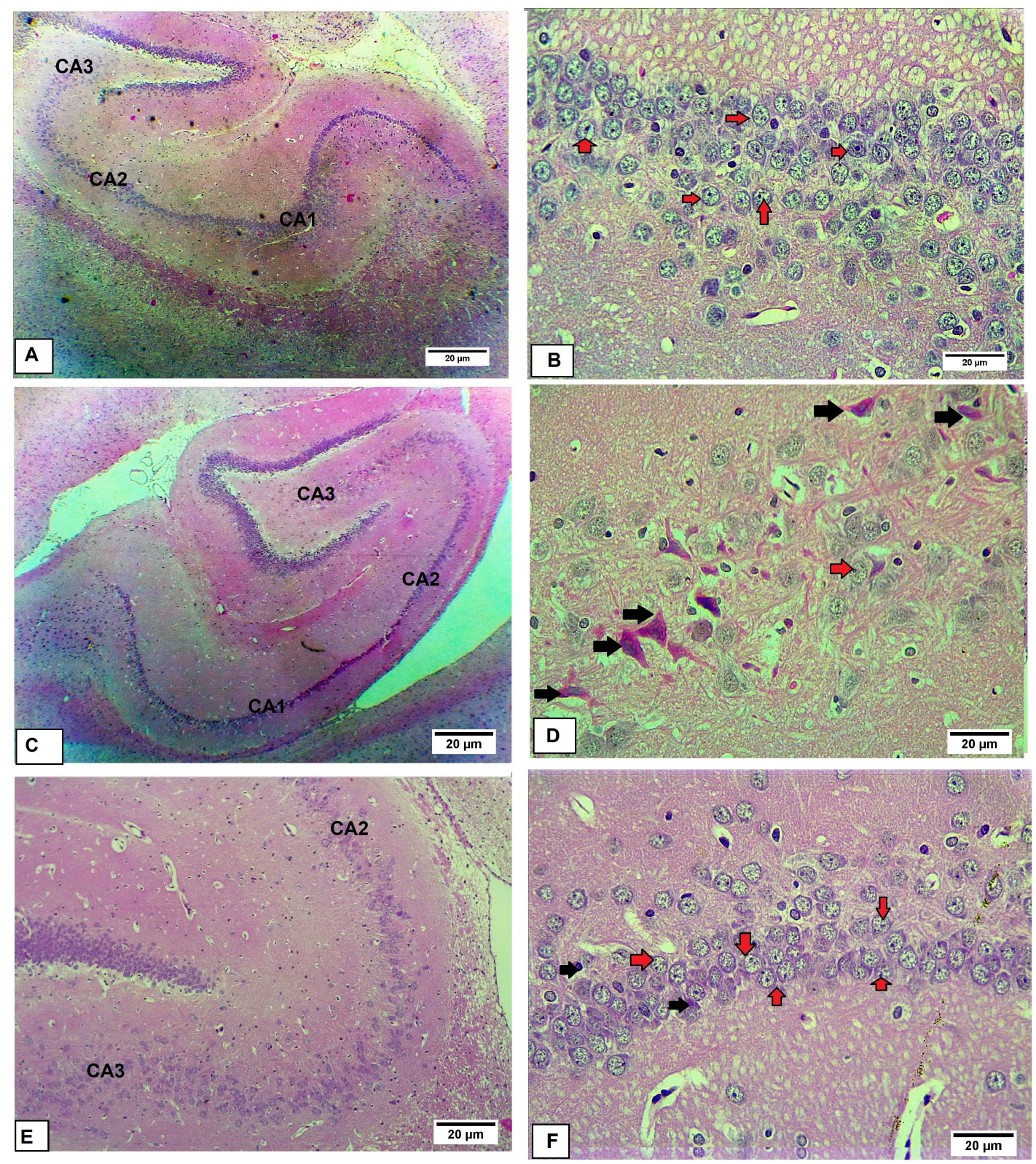

Fig. 4. Effects of VA on morphology of CA3 hippocampal region from different groups. Brain specimens showing (A) normal structure and thickness of hippocampal regions (CA3,2,1) (H\&E, 100×, control group) and (B) normal shape and distributions of neurons with round nuclei and prominent nucleoli (red arrows) in CA3 region (H\&E, 400 $\times$, control group). (C) Thinning and atrophy of CA3 region of hippocampus (H\&E, 100 $\times$, PTZ group). (D) Low number of round normal cells (red arrows) with dense pyknotic nuclei in died neurons in CA3 region (black arrows) (H\&E,400 $\times$, PTZ group). (E) Nearly normal structure and thickness of CA3 hippocampal region (H\&E, 200 $\times$, VA + PTZ group) and (F) increased number of normal neurons with round nuclei and prominent nucleoli (red arrows) with few died neurons (black arrows) in CA3 region (H\&E, 400×, VA + PTZ group).

\subsection{Correlations between IGF-1 and other studied parameters}

The expression of IGF-1 showed a negative significant correlation with the PTZ-induced epileptic seizure score ( $p=$ 0.0067) (Fig. 7A), a non-significant positive correlation with the seizure latency $(p=0.2401)$ (Fig. 7B). Also, the oxidative stress markers showed negative correlations between IGF-1 and MDA ( $p=0.0016)$ (Fig. 7C), and positive correlation between IGF-1 and CAT enzyme activity $(p<0.0001)$ and TAC $(p<0.0001)$ (Fig. 7D-E respectively). Moreover, there is a negative correlation between IGF-1 and GFAP $(p<0.0001)$
(Fig. 7F), while the expression of $\mathrm{Nrf} 2$ and $\mathrm{HO}-1$ showed positive correlations with IGF-1 and Nrf2 and HO-1 ( $p=0.0026$, 0.0172 respectively) (Fig. $7 \mathrm{G}-\mathrm{H}$ ).

\section{Discussion}

With an alternate day PTZ injection for two weeks, our study brought about a substantial increase in the PTZinduced seizures, the GFAP expression, and the extent of neuronal loss. Also, PTZ worsened the oxidative state and decreased the IGF-1 level with insignificant changes in 

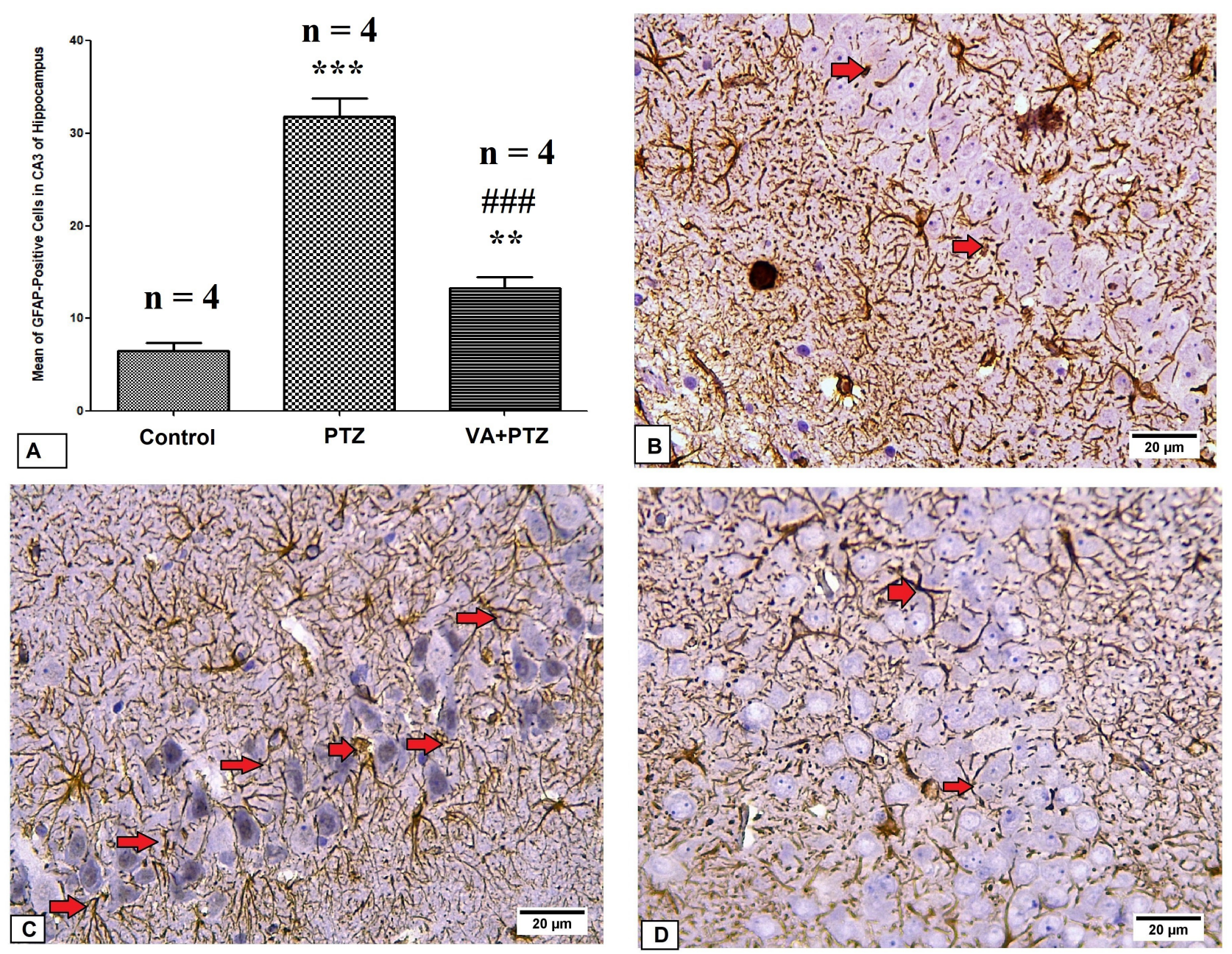

Fig. 5. Effects of VA on GFAP (marker of astrocytes and astrocytosis) expression in CA3 hippocampal region. (A) Represents the mean of GFAPpositive cells per high power field (HPF) in the CA3 region in different groups. Brain specimens from (B) normal control group shows normal expression (red arrows) of GFAP in CA3 region of hippocampus $(400 \times)$. (C) PTZ group show marked GFAP expression (red arrows) in CA3 hippocampal region (astrocytosis) $(400 \times)$ ). (D) VA + PTZ group show decreased GFAP expression (red arrows) in CA3 hippocampal region. One-way ANOVA with Tukey posthoc test, results were represented as mean \pm SEM. ${ }^{* * *}$ significant vs control group $p<0.0001{ }^{* *}$ significant vs control group $p<0.01$ and ${ }^{\# \# \#}$ significant vs PTZ group $p<0.0001 . \mathrm{n}=$ number of rats per group.

the Nrf2 and HO-1. Moreover, vanillic acid exhibited an antiepileptic and a neuroprotective role, since it markedly demoted the PTZ-induced seizures score, neuronal loss, and astrocytosis. Additionally, vanillic acid amended the oxidative state and elevated the Nrf2, HO-1, and IGF-1 expression.

In our study, high scored PTZ-induced seizures with a shortened latency and an extensive neuronal loss in the hippocampal region portrayed the existence of epileptogenesis. Also, PTZ mounted the GFAP expression signifying an expanded territory of astrocytes. A two weeks daily administration of $50 \mathrm{mg} / \mathrm{kg}$ vanillic acid decreased the score of PTZinduced seizures, neuronal loss, and astrocytosis and prolonged the seizures latency. The apparent neuroprotective role of vanillic acid has been captured in various neurological disorders. Ullah et al. [29] reported that a $30 \mathrm{mg} / \mathrm{kg}$ vanillic acid protected against lipopolysaccharide-induced neuroin- flammation, amyloidogenesis, memory deficit, and neurodegeneration in mice brain with a hopeful role in Alzheimer's disease. Khoshnam et al. [30] used a transient bilateral common carotid artery occlusion model followed by reperfusion with a 14 days vanillic acid administration prior to the hypoperfusion. Their study revealed a protecting outcome of vanillic acid in ischemia reperfusion-induced injury in a rat brain since it diminished reactive hyperemia and improved the disruption of blood brain barrier (BBB). Also, in a rotenone-induced Parkinson's disease rat model, Sharma et al. [31] displayed a protective effect of co-treatment of vanillic acid and levodopa-carbidopa, motor defects, and oxidative stress-induced neuronal damage indicating possible neuroprotection in Parkinson's disease. In consistence with the results of the current study, Nesterkina, et al. [32] reported an anticonvulsant effect of vanillic acid against PTZ-induced 

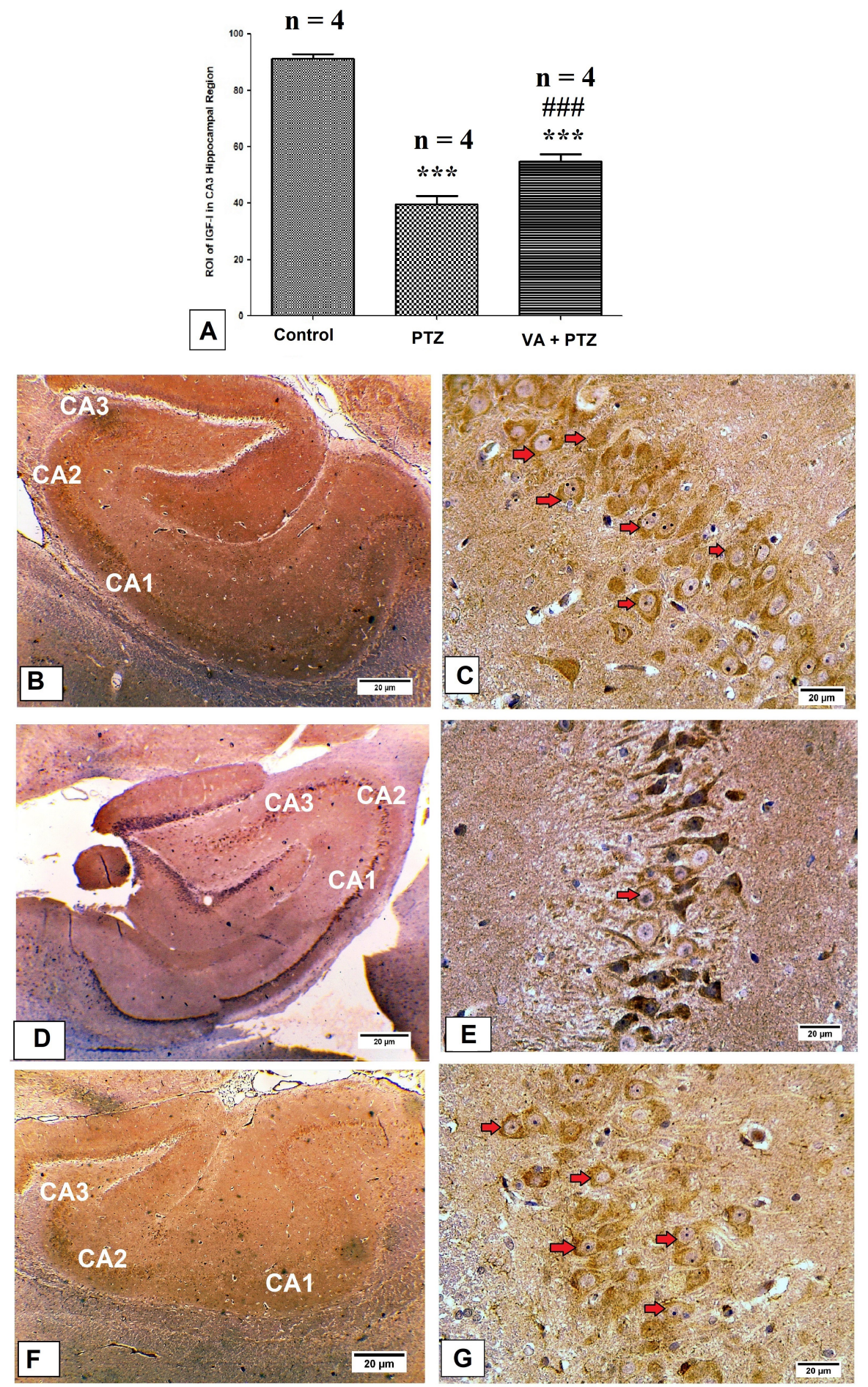

Fig. 6. Effects of Stevia on IGF-1 expression in CA3 hippocampal region by immunohistochemistry. (A) Represents the mean area of interest (ROI) of IGF-1 expression per high power field (HPF) in different groups. Brain specimens from normal control group show (B) different regions of hippocampus (CA1, CA2, and CA3) at low power $(100 \times)$. (C) Marked membranous expression (red arrows) of IGF-1 in CA3 region of hippocampus (400×). Brain specimens from PTZ group show (D) different regions of hippocampus (CA1, CA2, and CA3) at low power (100X). (E) mild membranous IGF-1 expression (red arrows) in CA3 hippocampal region (400X) and brain specimens from VA + PTZ group show (F) different regions of hippocampus (CA1, CA2, and CA3) at low power $(100 \times)$. (G) Moderate membranous IGF-1 expression (red arrows) in CA3 hippocampal region. One-way ANOVA with Tukey posthoc test, results were represented as mean $\pm \mathrm{SEM}$. ${ }^{* * *}$ significant vs control group $p<0.0001$ and ${ }^{\# \# \#}$ significant vs $\mathrm{PTZ}$ group $p<0.0001 . \mathrm{n}=$ number of rats per group. 

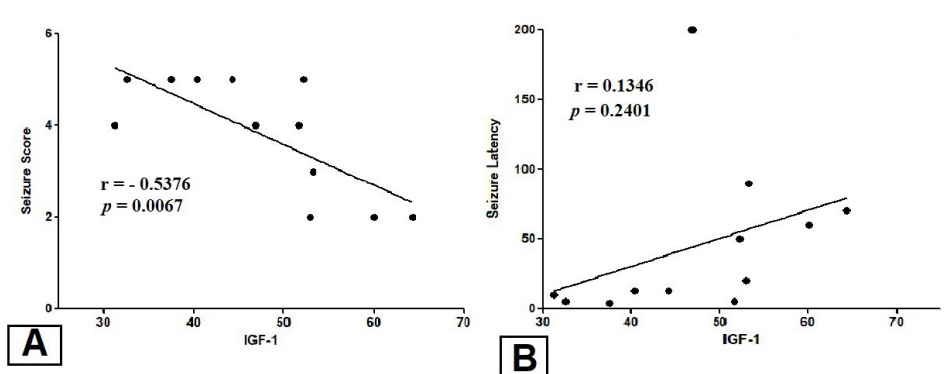

B
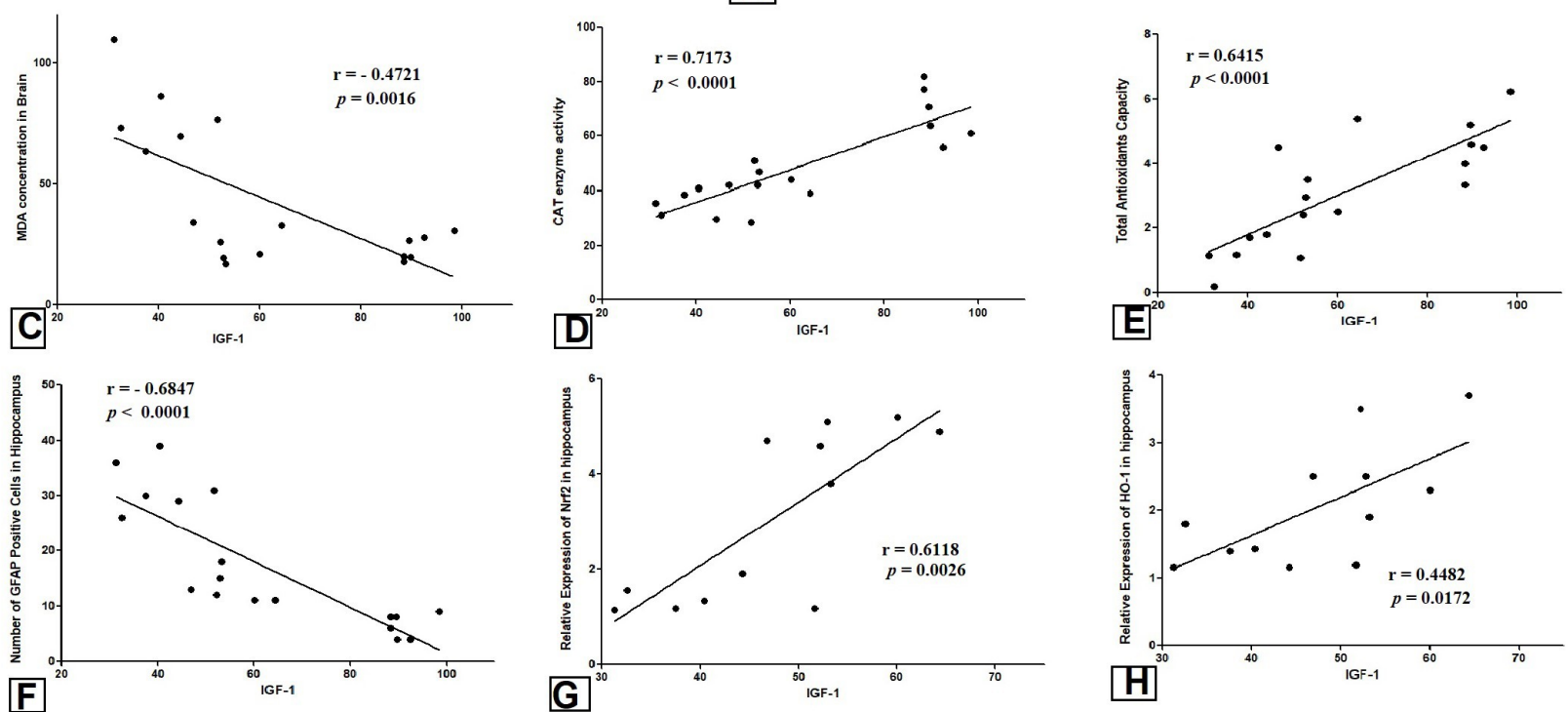

Fig. 7. Pearson correlations of IGF-1 expression. (A) Seizure score. (B) Seizure latency. (C) MDA. (D) Catalase enzyme activity. (E) Total antioxidant capacity. (F) GFAP. (G) Nrf2. (H) HO-1. $\mathrm{r}=$ correlation coefficient, $p=$ probability significance ( $p<0.05$ is considered significant).

seizures by minimum effective doses inducing clonic-tonic convulsions and tonic extension and against maximal electroshock induced seizures in mice. However, to the best of our knowledge, our study exclusively offers a novel dimension to the neuroprotective role of vanillic acid. We present vanillic acid not only as an anticonvulsant but also as a potential antiepileptic since it reduced the seizure score, neuronal loss, astrocytosis, and prolonged seizure latency.

Astrocytosis has a crucial role in the process of epileptogenesis intertwined with oxidative stress which is a key hallmark in epilepsy. Puttachary et al. [33] mentioned that reactive astrocytosis is accompanied by hyperexcitability due to the down-regulation of glutamine synthase enzyme resulting in high levels of un-metabolized glutamate. Moreover, this hyperexcitability results in the overproduction of free radicals overwhelming the antioxidant capacity and causing oxidative stress. This is well consistent with our findings since PTZ exhibited a high GFAP level, a marker for astrocytosis, and high oxidative stress markers. In the current study, vanillic acid reduced the GFAP expression and the oxidative stress markers. The neuroprotective effect of vanillic acid demonstrated in our study seems to be owing to its antioxidant properties and slowing down astrocytosis. This is in harmony with Amin et al. [24] who mentioned that $30 \mathrm{mg} / \mathrm{kg}$ vanillic acid administration for 3 weeks after $\mathrm{A} \beta_{1-42}$-injection improved the ox- idative state and attenuated astrocytosis in a mice model of Alzheimer's disease.

$\mathrm{Nrf} 2$ is a transcription factor that promotes the production of many antioxidant enzymes including glutathione peroxidases and HO-1 [13]. In the current study, a $50 \mathrm{mg} / \mathrm{kg}$ alternate day PTZ injection for 14 days showed an insignificant change in the expression of Nrf2 and HO-1 between the PTZ and the controls. This is consistent with Wang et al. [14] who used repeated administration of sub-convulsive electric stimulation for a 15 days amygdala kindling rat model. On the other hand, Li et al. [34] displayed a lowered expression of Nrf2 and HO-1 with a daily $37 \mathrm{mg} / \mathrm{kg}$ PTZ for 40 days in mice. The difference might emerge from the fact that Nrf2 is activated in epilepsy after oxidative stress as an endogenous adaptive mechanism to protect against the neuronal oxidant insult [14]. Vanillic acid upregulated the Nrf2/HO-1 expression which is in line with its antioxidant effect. The up-regulation of Nrf2/HO-1 expression has been a well-investigated notion as a potential therapeutic target for epilepsy [13]. Chen et al. [15] displayed amelioration of glutamate-induced oxidative damage by activating Nrf2/HO1 Signaling Pathway in HT22 Cells. Moreover, Amin et al. [24] showed a neuroprotective role of vanillic acid through Nrf2/HO-1 up-regulation in a mice model of Alzheimer's disease. So, up to the best knowledge, our study exclu- 
sively shows a neuroprotective role for vanillic acid in a PTZinduced epilepsy model through up-regulation of $\mathrm{Nrf2} / \mathrm{HO}-$ 1. However, we did not measure the expression of $\mathrm{Nrf} 2 / \mathrm{HO}-$ 1 at the level of protein by western blotting which is considered as a limitation of the current study to be considered in further studies.

IGF-1 enhances neuronal differentiation, maturation, survival, and axon growth [16]. This is well depicted in our findings since vanillic acid elevated the PTZ-induced lowered levels of IGF-1. Also, there was a positive correlation between IGF-1 and Nrf2 and a negative one between IGF1 and astrocytosis, neuronal loss, seizures score, and oxidative stress markers. Our hypothesis of the cross-talk between the two pathways of IGF-1 and Nrf2/HO-1 has been postulated in various studies. Mahran [35] reported an improvement of cisplatin-induced nephrotoxicity through growth hormone administration that increased IGF-1 expression along with up-regulation of Nrf-HO1 proposing IGF-1 as a mediator for Nrf2/HO-1 which eventually opposes the oxidative damage in renal cells induced by cisplatin. Bailey-Downs et al. [36] showed that IGF-1 deficiency impaired the vascular antioxidant responses by impairing Nrf-2 expression and its target genes. Also, Wang et al. [37] showed that IGF-1 treatment promoted the nuclear translocation of $\mathrm{Nrf} 2$, and up regulated the expression of its downstream gene (HO-1) in an Alzheimer's disease model with improvement in the oxidative state. Moreover, PI3-K inhibition abolished the protective effect of IGF- 1 on $\beta$-amyloid-induced ROS generation. This suggests that IGF-1 protects SH-SY5Y cells against $\beta$-amyloid induced cell injury by scavenging ROS via the PI3K/Akt-Nrf2 signaling pathway. Furthermore, Amin, et al. [24] showed that daily $30 \mathrm{mg} / \mathrm{kg}$ vanillic acid for 3 weeks improved oxidative stress markers, Nrf2, HO-1, Akt, and Gsk levels in mice brain homogenates after $\beta$-amyloid-induced oxidative stress. Although, the strongly suggested neuroprotective role of vanillic acid in PTZ-induced epilepsy in our study, a further clarification is needed concerning a more specific approach towards the involved signaling pathways such as PI3K/Akt pathway. Also, our study addressed a correlation between IGF-1, oxidative stress, Nrf-2 and astrocytosis which could open new perspectives in the study of the process of epileptogenesis. These potential novel perspectives require further studies.

\section{Conclusions}

In conclusion, vanillic acid demonstrated a neuroprotective and an anti-epileptic effect against PTZ-induced epilepsy. This outcome might be due to suppression of PTZinduced astrocytosis, and oxidative stress. Vanillic acid seems to act through up regulation of IGF-1 and Nrf2. Moreover, the potential cross-talk between IGF-1 and Nrf2 could share in the neuroprotective and the anti-epileptic effect of vanillic acid in a PTZ-kindled rat model.

\section{Author contributions}

MAE, AY, AMH, EME and MAA conceived and designed the study. MAE, SS, WO, AMH performed all behavioral experiments and conducted biochemical and molecular experiments. AMH, SS, WO, EME, MAA, and WAA collected the data and performed statistical analysis. MAE, AY, EME, WO, AY, SS and AMH wrote the manuscript. All authors reviewed and approved the final draft.

\section{Ethics approval and consent to participate}

The IRB-committee approved all experimental procedures (code \#R.20.11.1098.r).

\section{Acknowledgment}

The authors would also like to express their gratitude to King Khalid University, Saudi Arabia, for providing administrative and technical support.

\section{Funding}

The authors would also like to express their gratitude to the Deanship of Scientific Research at King Khalid University, Saudi Arabia for funding this project through the General Research Program (grant \# G.R.P/100/41).

\section{Conflict of interest}

The authors declare no conflict of interest.

\section{Data availability statement}

The data will be available on reasonable request.

\section{References}

[1] Falco-Walter J. Epilepsy-Definition, Classification, Pathophysiology, and Epidemiology. Seminars in Neurology. 2020; 40: 617623.

[2] Noebels J, Avoli M, Rogawski M, Olsen R, Delgado-Escueta A. Cognitive and behavioral co-morbidities of epilepsy. Jasper's Basic Mechanisms of the Epilepsies. 4th ed. National Center for Biotechnology Information: Bethesda, MD. 2012.

[3] Picot M, Baldy-Moulinier M, Daurès J, Dujols P, Crespel A. The prevalence of epilepsy and pharmacoresistant epilepsy in adults: a population-based study in a Western European country. Epilepsia. 2008; 49: 1230-1238.

[4] Lowenstein DH. Pathways to discovery in epilepsy research: rethinking the quest for cures. Epilepsia. 2008; 49: 1-7.

[5] Samokhina E, Samokhin A. Neuropathological profile of the pentylenetetrazol (PTZ) kindling model. Journal of Neuroscience. 2018; 128: 1086-1096.

[6] Martinc B, Grabnar I, Vovk T. The role of reactive species in epileptogenesis and influence of antiepileptic drug therapy on oxidative stress. Current Neuropharmacology. 2012; 10: 328-343.

[7] Lugrin J, Rosenblatt-Velin N, Parapanov R, Liaudet L. The role of oxidative stress during inflammatory processes. Biological Chemistry. 2014; 395: 203-230.

[8] Vezzani A, French J, Bartfai T, Baram TZ. The role of inflammation in epilepsy. Nature Reviews. Neurology. 2011; 7: 31-40.

[9] Santos IMS, Tomé ADR, Saldanha GB, Ferreira PMP, Militão GCG, Freitas RMD. Oxidative stress in the hippocampus during experimental seizures can be ameliorated with the antioxidant ascorbic acid. Oxidative Medicine and Cellular Longevity. 2009; 2: 214-221. 
[10] Diniz TC, Silva JC, Lima-Saraiva SRGD, Ribeiro FPRDA, Pacheco AGM, de Freitas RM, et al. The Role of Flavonoids on Oxidative Stress in Epilepsy. Oxidative Medicine and Cellular Longevity. 2015; 2015: 171756.

[11] Kiasalari Z, Khalili M, Shafiee S, Roghani M. The effect of Vitamin $E$ on learning and memory deficits in intrahippocampal kainateinduced temporal lobe epilepsy in rats. Indian Journal of Pharmacology. 2016; 48: 11-14.

[12] Hussein AM, Adel M, El-Mesery M, Abbas KM, Ali AN, Abulseoud OA. L-Carnitine Modulates Epileptic Seizures in Pentylenetetrazole-Kindled Rats via Suppression of Apoptosis and Autophagy and Upregulation of Hsp70. Brain Sciences. 2018; 8: 45.

[13] Carmona-Aparicio L, Pérez-Cruz C, Zavala-Tecuapetla C, Granados-Rojas L, Rivera-Espinosa L, Montesinos-Correa H, et al. Overview of Nrf2 as Therapeutic Target in Epilepsy. International Journal of Molecular Sciences. 2015; 16: 18348-18367.

[14] Wang W, Wu Y, Zhang G, Fang H, Wang H, Zang H, et al. Activation of $\mathrm{Nrf2}$-are signal pathway protects the brain from damage induced by epileptic seizure. Brain Research. 2014; 1544: 54-61.

[15] Chen X, Bao G, Liu F. Inhibition of USP15 Prevent GlutamateInduced Oxidative Damage by Activating Nrf2/HO-1 Signaling Pathway in HT22 Cells. Cellular and Molecular Neurobiology. 2020; 40: 999-1010.

[16] Wei M, Dong L, Zhang H, Teng Z, Wang X, Yan Z, et al. Review of Insulin-Like Growth Factor 1 Signaling Pathway and its Role in Protection against Brain Diseases. Translational Neuroscience and Clinics. 2017; 3: 237-245.

[17] Logan S, Pharaoh GA, Marlin MC, Masser DR, Matsuzaki S, Wronowski B, et al. Insulin-like growth factor receptor signaling regulates working memory, mitochondrial metabolism, and amyloid- $\beta$ uptake in astrocytes. Molecular Metabolism. 2018; 9: 141-155.

[18] Riis S, Murray JB, O'Connor R. IGF-1 Signalling regulates mitochondria dynamics and turnover through a conserved GSK-3 $\beta$ Nrf2-BNIP3 pathway. Cells. 2020; 9: 147.

[19] Kumar R, Sharma P, Mishra PS. Vanillin Derivatives Showing Various Biological Activities. Journal of Cheminformatics. 2012; 43.

[20] Soobrattee MA, Neergheen VS, Luximon-Ramma A, Aruoma OI, Bahorun T. Phenolics as potential antioxidant therapeutic agents: Mechanism and actions. Mutation Research. 2005; 579: 200-213.

[21] Calixto-Campos C, Carvalho TT, Hohmann MSN, Pinho-Ribeiro FA, Fattori V, Manchope MF, et al. Vanillic Acid Inhibits Inflammatory Pain by Inhibiting Neutrophil Recruitment, Oxidative Stress, Cytokine Production, and NFxB Activation in Mice. Journal of Natural Products. 2015; 78: 1799-1808.

[22] Kumar S, Prahalathan P, Raja B. Antihypertensive and antioxidant potential of vanillic acid, a phenolic compound in L-NAMEinduced hypertensive rats: a dose-dependence study. Redox Report. 2011; 16: 208-215.

[23] Tsuda H, Uehara N, Iwahori $Y$, Asamoto M, Ligo M, Nagao M, et al. Chemopreventive Effects of $\beta$-Carotene, $\alpha$-Tocopherol and Five Naturally Occurring Antioxidants on Initiation of Hepatocarcinogenesis by 2-Amino-3-methylimidazo[4,5- $\mathrm{f}]$ qumoline in the Rat. Japanese Journal of Cancer Research. 1994; 85: 1214-1219.
[24] Amin FU, Shah SA, Kim MO. Vanillic acid attenuates A $\beta \_1-42$ induced oxidative stress and cognitive impairment in mice. Scientific Reports. 2017; 7: 40753.

[25] Hussein AM, Eldosoky M, El-Shafey M, El-Mesery M, Ali AN, Abbas KM, et al. Effects of metformin on apoptosis and $\alpha$-synuclein in a rat model of pentylenetetrazole-induced epilepsy. Canadian Journal of Physiology and Pharmacology. 2019; 97: 37-46.

[26] Racine RJ. Modification of seizure activity by electrical stimulation. II. Motor seizure. Electroencephalography and Clinical Neurophysiology. 1972; 32: 281-294.

[27] Zahran MH, Hussein AM, Barakat N, Awadalla A, Khater S, Harraz A, et al. Sildenafil activates antioxidant and antiapoptotic genes and inhibits proinflammatory cytokine genes in a rat model of renal ischemia/reperfusion injury. International Urology and Nephrology. 2015; 47: 1907-1915.

[28] Hussein AM, Abbas KM, Abulseoud OA, El-Hussainy EMA. Effects of ferulic acid on oxidative stress, heat shock protein 70 , connexin 43 , and monoamines in the hippocampus of pentylenetetrazole-kindled rats. Canadian Journal of Physiology and Pharmacology. 2017; 95: 732-742.

[29] Ullah R, Ikram M, Park TJ, Ahmad R, Saeed K, Alam SI, et al. Vanillic Acid, a Bioactive Phenolic Compound, Counteracts LPSInduced Neurotoxicity by Regulating c-Jun N-Terminal Kinase in Mouse Brain. International Journal of Molecular Sciences. 2020; 22: 361.

[30] Khoshnam SE, Farbood Y, Fathi Moghaddam H, Sarkaki A, Badavi M, Khorsandi L. Vanillic acid attenuates cerebral hyperemia, blood-brain barrier disruption and anxiety-like behaviors in rats following transient bilateral common carotid occlusion and reperfusion. Metabolic Brain Disease. 2018; 33: 785-793.

[31] Sharma N, Khurana N, Muthuraman A, Utreja P. Pharmacological evaluation of vanillic acid in rotenone-induced Parkinson's disease rat model. European Journal of Pharmacology. 2021; 903: 174112.

[32] Nesterkina M, Fedorova E, Boyko Y, Kravchenko I. Vanillin derivatives affecting the central and peripheral nervous system. 4th International Electronic Conference on Medicinal Chemistry. Sponsored by Pharmaceuticals: Ukraine. 2018.

[33] Puttachary S, Sharma S, Stark S, Thippeswamy T. Seizure-induced oxidative stress in temporal lobe epilepsy. BioMed Research International. 2015; 2015: 745613.

[34] Li D, Bai X, Jiang Y, Cheng Y. Butyrate alleviates PTZ-induced mitochondrial dysfunction, oxidative stress and neuron apoptosis in mice via Keap1/Nrf2/HO-1 pathway. Brain Research Bulletin. 2021; 168: 25-35.

[35] Mahran YF. New insights into the protection of growth hormone in cisplatin-induced nephrotoxicity: the impact of IGF-1 on the Keap1-Nrf2/HO-1 signaling. Life Sciences. 2020; 253: 117581.

[36] Bailey-Downs LC, Mitschelen M, Sosnowska D, Toth P, Pinto JT, Ballabh P, et al. Liver-specific knockdown of IGF-1 decreases vascular oxidative stress resistance by impairing the Nrf2-dependent antioxidant response: a novel model of vascular aging. Biological Sciences and Medical Sciences. 2012; 67: 313-329.

[37] Wang Z, Xiong L, Wang G, Wan W, Zhong C, Zu H. Insulinlike growth factor-1 protects SH-SY5Y cells against $\beta$-amyloidinduced apoptosis via the PI3K/Akt-Nrf2 pathway. Experimental Gerontology. 2017; 87: 23-32. 